\title{
Sensory Input Enhances Synaptogenesis of Adult-Born Neurons
}

\author{
Yoav Livneh, ${ }^{1}$ Naomi Feinstein, ${ }^{1}$ Marguerita Klein, ${ }^{3}$ and Adi Mizrahi ${ }^{1,2}$ \\ ${ }^{1}$ Department of Neurobiology, Institute for Life Sciences and ${ }^{2}$ The Interdisciplinary Center for Neural Computation, The Hebrew University of Jerusalem, \\ Edmond J. Safra Campus, Givat Ram, Jerusalem 91904, Israel, and ${ }^{3}$ Department of Neurobiology, Duke University Medical Center, Durham, North Carolina \\ 27710
}

The adult mammalian brain maintains a prominent stem cell niche in the subventricular zone supplying new neurons to the olfactory bulb. We examined the dynamics of synaptogenesis by imaging the formation and elimination of clusters of a postsynaptic marker (PSD95), genetically targeted to adult-born neurons. We imaged in vivo adult-born periglomerular neurons (PGNs) during two phases of development, immaturity and maturity. Immature PGNs showed high levels of PSD95 puncta dynamics during 12-72 h intervals. Mature PGNs were more stable compared with immature PGNs but still remained dynamic, suggesting that synaptogenesis persists long after these neurons integrated into the network. By combining intrinsic signal and two photon imaging we followed PSD95 puncta in sensory enriched glomeruli. Sensory input upregulated the development of adult-born PGNs only in enriched glomeruli. Our data provide evidence for an activity-based mechanism that enhances synaptogenesis of adult-born PGNs during their initial phases of development.

Key words: adult-neurogenesis; synaptogenesis; olfactory bulb; in vivo imaging; periglomerular; sensory enrichment

\section{Introduction}

The adult mammalian brain maintains two unique neurogenic niches. One in the hippocampus and one in the subventricular zone (SVZ), which continuously supplies new neurons to the olfactory bulb (OB) (Altman, 1969; Alvarez-Buylla and GarciaVerdugo, 2002; Lledo et al., 2006). In the OB of rodents, adultborn neurons give rise to different subtypes of interneurons, primarily granule cells (GCs) and periglomerular neurons (PGNs). This unique population has been hypothesized to subserve a role in the network's plasticity, but this issue remains uncertain and under continuous investigation. It is clear that $\mathrm{OB}$ adult-born neurons are fully functional because they become indistinguishable from older neurons morphologically, chemo-anatomically and electrophysiologically (Carlén et al., 2002; Belluzzi et al., 2003; Carleton et al., 2003; Bagley et al., 2007; Whitman and Greer, 2007b). However, to understand their function, it is important to decipher their synaptic connectivity patterns within their respective networks, and to uncover how such patterns emerge (Toni et al., 2007).

Adult-born neurons' chemo-anatomical specificity as well as gross an anatomical location (i.e., layer specificity) has been recently shown to be determined by the precise birthplace of the

\footnotetext{
Received Aug. 28, 2008; revised Nov. 4, 2008; accepted Nov. 25, 2008.

This work was supported by Israeli Science Foundation Grant \#313-05 to A.M. A.M. is supported by a Career Development Award from the International Human Frontier Science Program Organization. We thank G. Raday and R. Eavry for assistance at early stages of this work and M. Belenky for advice regarding EM procedures. We thank N. Book-Melamed for technical assistance. We thank H. Spors for the analysis software of the intrinsic signals and members of the Mizrahi lab for discussions. We thank T. Kosaka and B. Arenkiel for critically commenting on early versions of this manuscript. Finally, we thank the late L. C. Katz for his kind support during early stages of this work Correspondence should be addressed to Adi Mizrahi at the above address. E-mail: mizrahia@cc.huji.ac.il. DOI:10.1523/JNEUROSCI.4105-08.2009

Copyright $\odot 2009$ Society for Neuroscience $\quad 0270-6474 / 09 / 290086-12 \$ 15.00 / 0$
}

progenitors as well as by the transcription factor expression profiles therein (Hack et al., 2005; Waclaw et al., 2006; Merkle et al., 2007). However, synaptic connectivity patterns might be determined by factors other than neuronal chemo-anatomical type. Moreover, the mechanisms that shape the formation of this synaptic connectivity are unknown, leaving numerous questions open. For example, once early phases of development are completed, does synaptogenesis cease? If not, to what extent does synaptogenesis remain a plastic process? What might be the mechanisms that control synapse formation during integration into the network?

One possibility is that the mechanisms that determine synaptogenesis are indeed completely intrinsic to the neurons, similar to the mechanisms of chemo-anatomical and layer specificity (Kelsch et al., 2007; Merkle et al., 2007). Conversely, another possibility is that local cues, such as levels of sensory input will shape adult-born neurons' development during the period of synaptic integration, as indicated by manipulations on neuronal excitability in hippocampal neurons (Ge et al., 2006; Tashiro et al., 2006).

Here, we set out to study the mechanisms that regulate synaptogenesis of adult-born PGNs, in vivo. Using in vivo two-photon imaging, we studied the structural dynamics of dendrites and putative synapses of adult-born PGNs, during their initial phases of synaptic integration into the network and after complete integration. We first describe the basic structural maturation pattern of putative synapses on PGN dendrites, which is a dynamically rich process. We also show that putative synapses of PGNs remain dynamic long after integration into the network, albeit at a lower rate. Combining intrinsic signal imaging, odor enrichment and two photon imaging, we show that sensory input accelerated synaptogenesis and dendritic development of PGNs only in en- 
riched loci. Together, we describe the developmental process of synaptogenesis of adult-born PGNs and show that its initial phases are regulated by sensory input.

\section{Materials and Methods}

Animals. We used male and female BALB/C mice or in a few cases M71GFP mice (Bozza et al., 2002) ( $n=67$ postnatal day 55-80). Of the 67 mice, 51 mice were used for in vivo imaging and for fixed tissue analysis, 6 mice were processed for TEM and 10 mice were used for ISI calibration and experiments. M71-GFP mice express GFP in a subset of ORNs serving here as in vivo morphological indicators that the $\mathrm{OB}$ is healthy. There were no evident differences between BALB/C and M71-GFP mice. For TEM analysis we also used a single YFP mouse (Feng et al., 2000) line-H. Animal care and experiments were approved by the Hebrew University Animal Care and Use Committee.

Lentiviral vectors. For synaptic labeling we used lenti-PSD95-GFP controlled by the Elongation-Factor $-1-\alpha$ promoter. We first cloned a "PSD95-GFP-IRES-DsRed" into pENTR2B vector (Invitrogen). Infective viri were then prepared by TranzVector (Tranzyme). Whereas the plasmid also contained a DsRed gene separated by an internal ribosome entry site, DsRed expression was too low in these neurons to use for the in vivo imaging experiments. DsRed could not be detected even in fixed tissue, or with immunohistochemical amplification with streptavidin (data not shown). It remains possible that some dendrites which lack puncta might be missed in our analysis. However, two results argue against a considerable bias. First, dendritic arbors of PSD95-GFP neurons are similar to those of GFP-labeled neurons (see supplemental Fig. 2, available at www.jneurosci.org as supplemental material). Second, we always observed a few dendrites that had cytoplasmic signal with no puncta. In all those cases, the cytoplasmic signal was diffused equally in proximal and distal dendritic endings and never fragmented.

For cytosolic GFP labeling, a commercial lentivirus (TranzVector, Tranzyme) containing the gene for GFP and controlled by the Elongation Factor-1- $\alpha$ promoter was used to transduce adult-born neurons. Virus titers were $>10^{8}$ transducing Us $/ \mathrm{ml}$.

Stereotaxic injection, two-photon imaging, and analysis. Lentivirus injections into the SVZ and imaging were performed as described earlier (Mizrahi, 2007). Mice were anesthetized by ketamine/xylazine $(0.1 \mathrm{mg}$ per g of body weight, i.p.). Additional anesthesia ( $1 \%$ isoflurane in $\mathrm{O}_{2}$, by inhalation) was supplied as needed. Injections were done stereotaxically using pressure (coordinates relative to Bregma: anterior- $1 \mathrm{~mm}$, lateral-1 $\mathrm{mm}$, depth $2.2 \mathrm{~mm}$; anterior- $0 \mathrm{~mm}$, lateral $-1.4 \mathrm{~mm}$, depth $1.6 \mathrm{~mm}$ ). Each mouse received a single or a double injection on the left and/or right SVZ (estimated injection volume is $\sim 0.5 \mu \mathrm{l}$ ). The approximate success rate in lentivirus injections was $\sim 70 \%$ ( $40 / 57$ mice, used for in vivo imaging, fixed tissue analysis and TEM; see above). The total number of labeled neurons (GCs + PGNs) varied between injections but always resulted in much larger numbers of GCs compared with PGNs. We limited our in vivo experiments to nonspiny PGNs. In a few cases we also identified spiny neurons at the GL but these neurons were not studied in this work. A $0.1 \mathrm{~g}$ metal bar was glued to the skull for repositioning the animal's head under the microscope in consecutive imaging sessions (Mizrahi and Katz, 2003). After surgery, mice fully recovered and returned to the animal facility under normal housing conditions until imaging. The 12-57 day postinjection (d.p.i.) mice were taken for imaging. Imaging was performed in anesthetized freely breathing mice usually through thinned skull. We normally performed imaging via thinned bone because it proved more reliable for reidentification of the same synaptic puncta on consecutive imaging sessions. However, because imaging via thinned bone is limiting the depth of high contrast imaging, we also used craniotomies when cells were located in the deep glomerular layer (GL). We did not observe any significant differences in structural dynamics between imaging via thinned bone compared with imaging via a craniotomy [data not shown, but see (Xu et al., 2007) for longer imaging intervals].

Imaging was performed on an Ultima microscope (Prairie Technologies) equipped with a $40 \times(0.8 \mathrm{NA})$ IR-Achroplan water-immersion objective (Olympus). A femtosecond laser (Mai-Tai, Spectra Physics) was used to excite GFP at $900 \mathrm{~nm}$. Images $(512 \times 512$ pixels $)$ were acquired at $0.25 \mu \mathrm{m} /$ pixel resolution in the $\mathrm{XY}$ dimension and $0.9 \mu \mathrm{m}$ steps in the $\mathrm{Z}$ dimension.

Reconstructions were performed strictly on the raw data images. Reconstructions were performed manually from the complete three dimensional image stacks using Neurolucida (MicrobrightField). Quantitative parameters included the number of branch points per tree, total dendritic branch length (TDBL), number of puncta per cell and Sholl analysis (Sholl, 1953). A dendritic segment was defined as the part of a dendrite between two intersections, between an intersection and an ending, or a retracted part longer than $1 \mu \mathrm{m}$ (see below). For dendritic dynamics, only segments longer than $1 \mu \mathrm{m}$ were analyzed. Retracted dendritic segments were defined as being present in one imaging session, but not in the next imaging session. Stable dendritic segments were defined as being present in both imaging sessions. For puncta dynamics, only dendritic segments that remained stable in consecutive imaging sessions were analyzed. For display purpose only, image stacks were filtered (median filter), projected as maximum intensity projections or SD projections, and adjusted for contrast and brightness using ImageJ (http://rsb.info. nih.gov/ij/). Merged images from time-lapse series were aligned in ImageJ using the turboreg plugin (http://bigwww.epfl.ch/thevenaz/ turboreg/). Reconstruction reliability between two different independent reconstructers was $92.3 \%$; reconstruction reliability between two reconstructions of the same reconstructer was $95.7 \%$.

To validate that short imaging intervals do not hold substantial dynamics, we performed imaging experiments in time scale of minutes (see supplemental Fig. 6, available at www.jneurosci.org as supplemental material). Puncta and dendrites were generally stable with relatively few "new" and "lost" puncta or dendrites ( $n=3$ neurons). Furthermore, these experiments show that lost puncta are not simply puncta that underwent photobleaching, because almost all puncta were stable even during six imaging sessions, $\sim 20 \mathrm{~min}$ apart. We did not further investigate these short time scales for two reasons. First, mice under these conditions are continuously anesthetized during imaging, which may disrupt normal cytoskeletal dynamics. Second, our data show that a $12 \mathrm{~h}$ imaging interval is an adequate interval to study structural dynamics (see main text).

Immunohistochemistry and confocal microscopy. To obtain OB slices, mice were perfused transcardially with $0.9 \%$ saline followed by $4 \%$ formaldehyde and the brain was cryoprotected in $30 \%$ sucrose. OBs were sectioned coronally on a sliding microtome ( $40 \mu \mathrm{m}$ slices), washed in PBS and blocked for $1 \mathrm{~h}$ in 5\% normal goat serum and $0.3 \%$ Triton-X. Slices were incubated overnight with a primary antibody (in the blocking solution), washed in PBS, and then incubated for $3 \mathrm{~h}$ with a secondary antibody (in the blocking solution). Slices were then washed and mounted on microscope slides. The primary antibody used was mouse anti-VGLUT2 (1:500) from Millipore. The secondary antibody used was anti-mouse Cy5 (1:200) from Jackson ImmunoResearch. Confocal images were obtained using an Olympus FV-1000 confocal microscope.

Analysis, of PSD95-GFP puncta apposition to VGLUT2 labeling, was performed on complete three dimensional image stacks. Puncta were considered to be opposed to VGLUT2 labeling if they exhibited fluorescent colocalization (yellow instead of green) (see Fig. 2C), and were encompassed by VGLUT2 in all dimensions $(x, y$, and $z)$.

Statistical analyses. All statistical analyses were performed using SPSS software (SPSS). Nonparametric tests were used for small samples (Kruskal-Wallis test followed by Mann-Whitney test), whereas the Student's $t$ test was used for larger samples. Significances were accepted at $p=0.05$, except when the Bonferroni correction for multiple comparisons was applied.

Intrinsic signal imaging and analysis. Intrinsic signal imaging (ISI) of the dorsal surface of the bulb was performed using an Imager 3001 (Optical Imaging) via thinned bone. Before each experiment, the surface blood vessel pattern was acquired under green light illumination (546 $\mathrm{nm})$. Light reflectance from the surface of the OB (630 nm wavelength light illumination) was captured using a CCD camera (Dalsa 1M60P). Images were acquired with a spatial resolution of $\sim 20 \mu \mathrm{m} /$ pixel (full frames were $1024 \times 1024$ pixels and binned $(3 \times 3)$ for analysis). IS maps were analyzed off-line, using a Matlab-based software which was developed and kindly provided to us by H. Spors (Max Planck Institute of 
Biophysics, Frankfurt, Geremany). IS maps were created by subtracting the averaged response to pure oxygen from the averaged response to the odorants. Odorants were presented using a custom-made olfactometer (stimulus duration, $4 \mathrm{~s}$ ). Odor stimuli were composed of a methylbenzoate and butanal mixture (1:1, >99\% purity, $10 \mathrm{ppm}$ each diluted in mineral oil) and resulted in activation of several overlapping glomeruli. The surface blood vessel pattern was later used to align intrinsic signal images with two-photon images as described previously (Mizrahi and Katz, 2003).

ISI images were first analyzed in a course manner (by signal threshold) to designate active or nonactive areas for reference during two-photon imaging (see Fig. 5B). A more detailed analysis was used at the exact location of all the imaged PGNs. Specifically, each PGN location was examined based on its time course and signal strength. Active areas exhibited a time course that was locked to the stimulus onset and showed a decrease in intrinsic signal $\left(>2 \times 10^{-4}\right)$. Nonactive areas did not meet both conditions.

Odor enrichment. Odor-enriched mice were housed in their home cages with an "odor pot" hanging from the top of the cage. This odor pot contained $150 \mu \mathrm{l}$ of $20 \mathrm{ppm}$ odor mixture (methylbenzoate and butanal, 1:1 diluted in mineral oil) on a swab. The swab was replaced approximately every $24 \mathrm{~h}$. The smell of a freshly made odor pot was barely noticeable by us or other members of our lab. As a further control experiment, we verified that the ISI odor mapping by itself did not cause the morphological effect (supplemental Fig. 7, available at www.jneurosci. org as supplemental material).

Preembedding immunoelectron transmission microscopy. Mice were perfused transcardially using $300 \mathrm{ml}$ of $0.25 \%$ glutaraldehyde and $2 \%$ formaldehyde in $0.1 \mathrm{M} \mathrm{PB}, \mathrm{pH} 7.4$ at room temperature (RT), at a rate of $10 \mathrm{ml} / \mathrm{min}$. OBs were dissected out and first cut into $60 \mu \mathrm{m}$ coronal slices using a vibratome (Leica VT100). Vibratome sections were screened for labeled PGNs using standard fluorescent microscopy. Once the labeled PGNs were located, the slices were aligned and photographed (using a still camera), and a region corresponding to $\sim 5$ glomeruli was then isolated for further targeting of the cells. Isolated regions, normally containing one labeled PGN, were then processed for TEM separately.

To abolish endogenous peroxidases, the sections were incubated for 10 min in $0.015 \% \mathrm{H}_{2} \mathrm{O}_{2}$ (in PBS). Sections were cryoprotected in a solution of $15 \%$ sucrose $/ 5 \%$ glycerol in PBS for $20 \mathrm{~min}$ in RT and then in a solution of $30 \%$ sucrose $10 \%$ glycerol in PBS in $4^{\circ} \mathrm{C}$, overnight. Then, sections were frozen in liquid nitrogen-cooled isopentane, followed by liquid nitrogen and thawed in PBS. For immunolabeling, the sections were incubated in a blocking solution containing 5\% normal rabbit serum (NRS), $2 \%$ bovine serum albumin (BSA), $0.5 \%$ glycine, $0.5 \%$ lysine, $0.01 \%$ Triton X-100 and $0.13 \%$ sodium azide in PBS for $1 \mathrm{~h}$ (at RT). Slices were incubated overnight in an anti-GFP primary antibody (chicken anti-GFP 1:600, Millipore) in 2\% NRS and 0.01\% Triton X-100 in PBS, and then with a biotinylated secondary antibody (1:500 rabbit anti-chicken $\mathrm{F}\left(\mathrm{ab}^{\prime}\right)_{2}$ fragment, The Jackson Laboratory). Labeling was revealed using the avidin-biotin peroxidase complex (ABC Elite, Vector Laboratories) and 3,3'-diaminobenzidine tetrachloride. The reaction product was intensified and substituted with silver/gold particles as described by others (Belenky et al., 2003). Briefly, slices were incubated for $10 \mathrm{~min}$ at $60^{\circ} \mathrm{C}$ in a solution containing $2.6 \%$ hexamethylene tetramine, $0.2 \% \mathrm{AgNO}_{3}$, and $0.2 \%$ Borax, washed in $2 \%$ sodium acetate and incubated for $5 \mathrm{~min}$ in $0.05 \% \mathrm{HAuCl}_{4}$ at RT, washed in $2 \%$ sodium acetate and then incubated for $5 \mathrm{~min}$ in $3 \% \mathrm{Na}_{2} \mathrm{SO}_{3}$ at RT. The sections were then postfixed with $1 \%$ osmic acid and $1.5 \%$ potassium ferricyanide in $0.1 \mathrm{~m}$ cacodylate buffer, $\mathrm{pH} 7.4$, for $1 \mathrm{~h}$ in RT, dehydrated in a graded series of ethanol (30-100\%) and embedded in increasing concentrations (50-100\%) of Agar 100 resin (Agar Scientific). Once cured in a $60^{\circ} \mathrm{C}$ oven for $48 \mathrm{~h}$, ultrathin sections of the region, in which a labeled PGN was located, were sectioned using an LKB-3 ultramicrotome, collected onto thin bar 200\# cuprum grids, and then stained with saturated aqueous uranyl acetate and lead citrate solutions. Control sections were processed as described above except that the primary antibody was omitted. Control sections had no specific staining. Ultrathin sections were observed with a Tecnai 12 (Phillips) TEM equipped with MegaView II CCD camera and AnalySIS version 3.0 software (SoftImaging System). Slices from one mouse were processed as described, but without the intensification and substitution with silver/gold particles. These slices were used as a control to verify that the intensification and substitution with silver/gold particles did not cause any alterations in the tissue.

Postembedding immunoelectron microscopy. Mice were perfused transcardially using $300 \mathrm{ml}$ of $0.25 \%$ glutaraldehyde and $2 \%$ formaldehyde in $0.1 \mathrm{M} \mathrm{PB}, \mathrm{pH} 7.4$, or $4 \%$ formaldehyde in $0.1 \mathrm{M} \mathrm{PB}, \mathrm{pH} 7.4$ at room temperature (RT), at a rate of $10 \mathrm{ml} / \mathrm{min}$. OBs were dissected out and first cut into 60 or $400 \mu \mathrm{m}$ coronal slices using a vibratome (Leica VT100).

Cortex sections of YFP mice were then dehydrated in a graded series of ethanol and embedded in LRWhite (London Resin), as previously described (Micheva and Smith, 2007). In OB sections, this process greatly reduced the fluorescence and so we performed a more moderate dehydration process. OB Slices were dehydrated in a different graded series of ethanol (30-70\%) and then embedded in 100\% LRWhite. Once cured in a $50^{\circ} \mathrm{C}$ oven for $24 \mathrm{~h}$, fluorescence was examined in the LR-White block (supplemental Fig. $1 B, E$, available at www.jneurosci.org as supplemental material). Ultrathin sections of the region, in which labeled neurons were located, were thin-sectioned, collected onto formvar-coated 200\# nickel grids, and processed for postembedding immunolabeling. Grids were incubated in drops of reagent on Parafilm within a wet chamber and the various steps alternated with washes in drops of Tris-buffered saline (TBS) containing $20 \mathrm{~mm}$ Tris-base, $0.9 \% \mathrm{NaCl}, 0.5 \% \mathrm{BSA}, 0.5 \%$ Tween 20 and $0.13 \% \mathrm{NaN}_{3}, \mathrm{pH} 8.2$. This was followed by $1 \mathrm{~h}$ incubation in a blocker containing $5 \%$ normal goat serum (NGS) in TBS and incubation either for a few hours or overnight with the primary antibody (chicken anti-GFP 1:100; Millipore). After that, grids were incubated for $90 \mathrm{~min}$ with a $12 \mathrm{~nm}$ gold-conjugated secondary antibody (1:10 donkey antichicken, The Jackson Laboratory), and then washed in TBS. Sections were then stained with saturated aqueous uranyl acetate and lead citrate solutions. Control sections were processed as described above except that the primary antibody was omitted. Control sections had no specific staining.

\section{Results}

\section{Targeting synaptogenesis in adult-born neurons}

Our experimental strategy for labeling synapses of adult-born neurons was to express a GFP-tagged postsynaptic protein. We chose the postsynaptic density scaffold protein, PSD95, which is largely restricted to synapses and is a commonly used postsynaptic marker in vitro and in vivo (Washbourne et al., 2002; Ebihara et al., 2003; Niell et al., 2004). We used a lentivirus ("lenti-PSD95GFP") injected into the subventricular zone (SVZ) as the transducing system to selectively label adult-born neurons and synapses (Fig. $1 A)$. A single virus injection $(\sim 0.5 \mu \mathrm{l})$ typically labeled hundreds of neuronal progenitors which migrated into the OB. We focused our study on two experimental age groups. One age group was "immature" neurons (12-14 d.p.i.) corresponding to "stage 4" neurons (Petreanu and Alvarez-Buylla, 2002). This age group was chosen because it is known to be within a developmental phase in which synaptogenesis is at its peak (Belluzzi et al., 2003; Carleton et al., 2003; Whitman and Greer, 2007a). The second age group was "mature" adult-born neurons (40-60 d.p.i.), corresponding to fully integrated neurons.

At 12-14 d.p.i., labeled adult-born neurons were located throughout the OB. Neuronal progenitors were located in the rostral migratory stream (RMS) and had the morphology of migrating neuroblasts (i.e., elongated cell body with a leading neurite) with a diffuse fluorescent signal throughout their cytosol (Fig. $1 B$ ). Outside the RMS, neurons were scattered in the granule cell layer (GCL) and the glomerular layer (GL). In the GL, most neurons were periglomerular neurons (PGNs) extending dendritic trees into the immediate glomerular neuropile. PGN dendrites were studded with punctuate fluorescent signals corresponding to the PSD95-GFP protein aggregations (Fig. 1C). In the GCL, neurons had classical granule cell morphology. Fluores- 


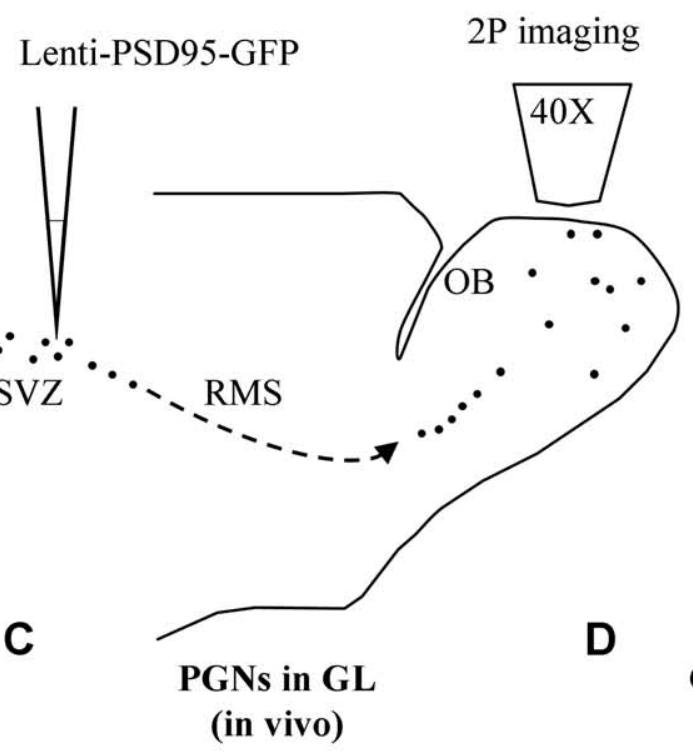

14 d.p.i

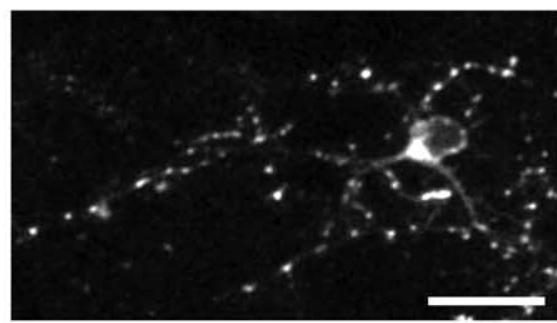

E

$$
41 \text { d.p.i }
$$
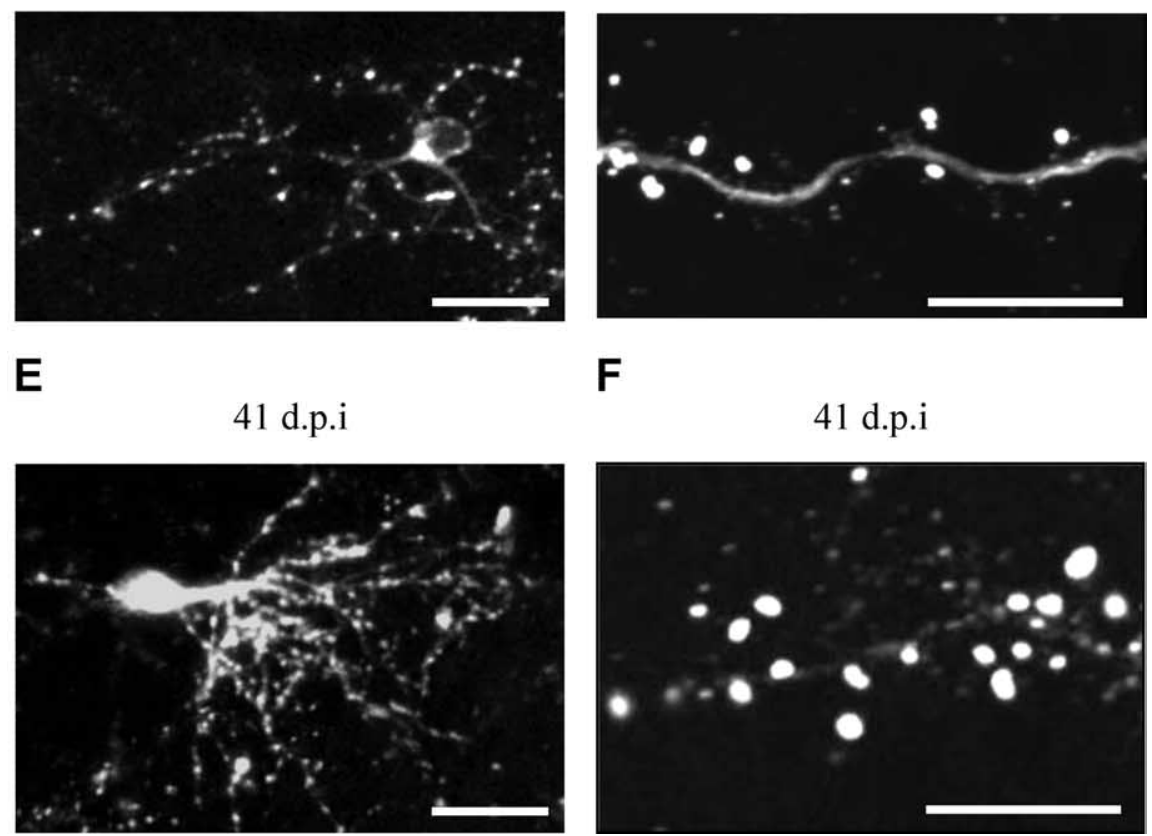

$\mathbf{F}$

\section{GC dendrites in EPL (fixed tissue)}

14 d.p.i

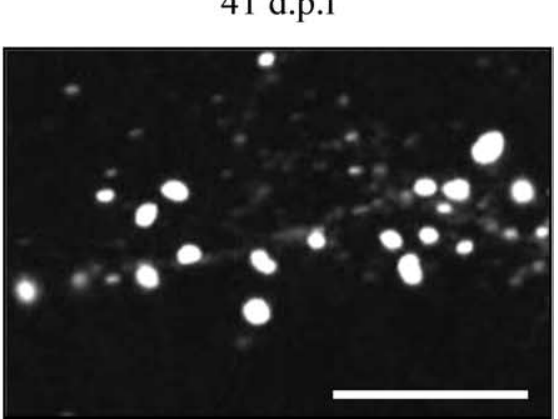

Figure 1. Targeting synapses of adult-born neurons using lenti-PSD95-GFP. $\boldsymbol{A}$, Schematic representation of the mouse brain and the experimental protocol. Lenti-PSD95-GFP injections were targeted to the subventricular zone (SVZ), and imaging was performed at different times after injections, using two-photon (2P) imaging. $\boldsymbol{B}$, Confocal micrograph of labeled neurons from the RMS, exhibiting the morphology of migrating neuroblasts. $\boldsymbol{C}$, In vivo two-photon micrograph of an immature PSD95-GFPexpressing PGN in the GL (14 d.p.i.). Notice that the dendritic trees are studded with punctuate fluorescent signals, corresponding to PSD95-GFP protein aggregations. D. Confocal micrograph of a dendritic segment from an immature PSD95-GFP-expressing GC in the GCL (14 d.p.i.). Notice that the PSD95-GFP signal is mainly in spine heads. $\boldsymbol{E}$, In vivo two-photon micrograph of a mature PSD95-GFP-expressing PGN in the GL (41 d.p.i.). $F$, Confocal micrograph of a dendritic segment from a mature PSD95-GFPexpressing $G C$ in the EPL (41 d.p.i.). All images are projections. Scale bars: $(\boldsymbol{B}, \boldsymbol{C}, \boldsymbol{E}) 20 \mu \mathrm{m} ;(\boldsymbol{D}, \boldsymbol{F}) 10 \mu \mathrm{m}$. spiny dendrite from a mature GC, both expressing PSD95-GFP, are shown in Figure $1, E$ and $F$, respectively. In all neurons, dendritic trees appeared as pale backbones compared with the fluorescence of the PSD95-GFP puncta. Although the vast majority of the labeled neurons were GCs, here we focused on the PGNs because they synapse locally in defined functionalanatomical units (i.e., the glomeruli), and their superficial location makes them more accessible for in vivo imaging.

Can PSD95-GFP puncta in PGN dendrites be used as proxy for synapses? Several results indicate that they can. First, to correlate between the fluorescent signal and actual synapses, we used immunotransmission electron microscopy (TEM). PSD95-GFP fluorescence was identified in coronal slices at low magnification. We then isolated a region corresponding to $\sim 5$ glomeruli and processed it for TEM with antibodies targeted to GFP (Fig. 2A). As expected from the highly sparse labeling, we had to screen hundreds of ultrathin sections to reveal a labeled synapse. However, once found, it provided unequivocal evidence that some puncta are indeed synapses. As depicted in Figure $2 B$, anti-GFP labeling appeared as an electron-dense grainy aggregate, mainly confined to the PSD (see more examples in supplemental Fig. 5, available at www.jneurosci.org as supplemental material). Second, to further validate this finding we used a different immuno-TEM approach, which requires less tissue processing and has advantages for immunostaining (Micheva and Smith, 2007). As expected, we found GFP immuno-staining confined to synapses in the EPL and GL (supplemental text and supplemental Fig. 1, available at www. jneurosci.org as supplemental material). Third, we performed immuno-staining against Vesicular Glutamate Transporter 2 (VGLUT2), one of the presynaptic markers in the glomerular layer, specific to olfactory receptor neurons (ORNs; Gabellec et al., 2007). In almost all examined neurons (10/11 immature neurons from 3 mice, and 10/10 mature neurons from 3 mice) a significant fraction of PSD95-GFP puncta appeared in close apposition to the VGLUT2 labeling (Fig. 2C). Quantification of these appositions in mature neurons showed that almost one-half of their cent signal was scattered throughout the neurons with more intense PSD95-GFP signal within spine heads in the external plexiform layer (EPL) (Fig. 1D). At $\sim 40$ d.p.i., adult born neurons were largely devoid of the RMS and restricted to the GCL and GL. Mature PGNs and GCs had a dendritic morphology similar to that we and others have described previously (Mizrahi, 2007; Whitman and Greer, 2007b). Examples of a mature PGN and a postsynaptic puncta were opposed to this presynaptic marker (46 $\pm 4 \%)$. Whereas appositions with VGLUT2 labeling represent only a subset of putative synapses (i.e., others being dendrodendritic synapses with Mitral and Tufted cells), it further demonstrates "correct" synaptic appositions of the PSD95-GFP signal. Finally, in spiny GCs, the PSD95-GFP signal is localized in spine heads; and, spines are good proxies for synapses (Knott et 
A

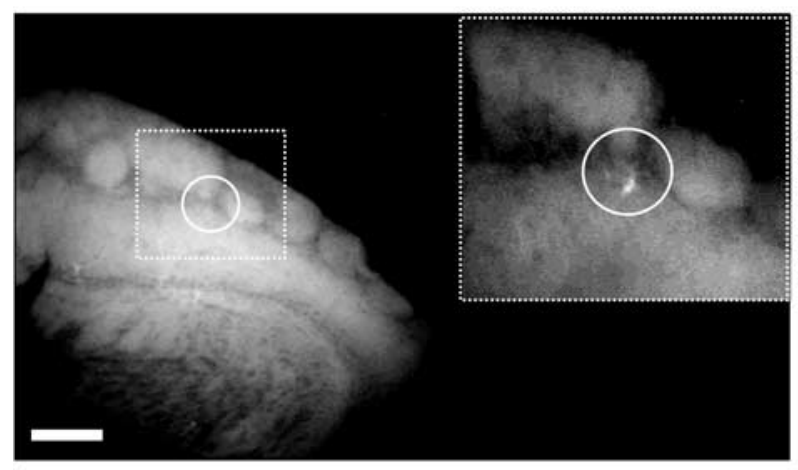

B

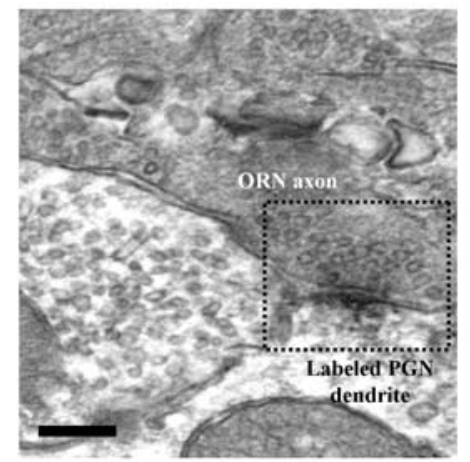

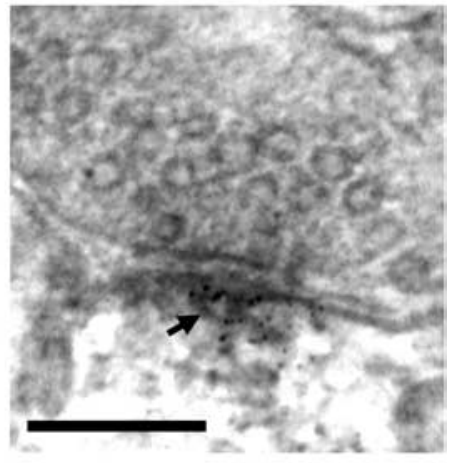

C
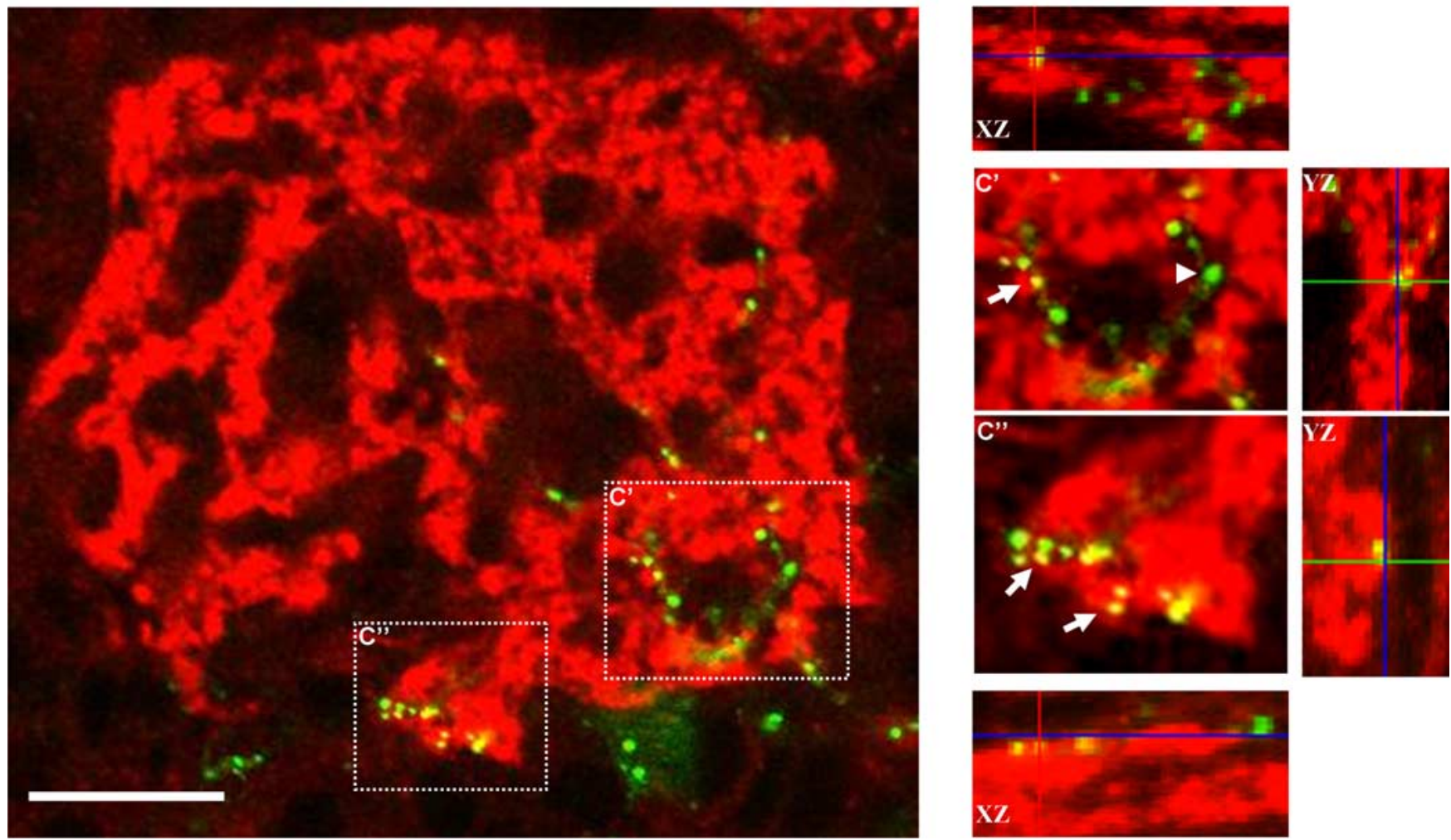

Figure 2. PSD95-GFP puncta as proxy for synapses. $\boldsymbol{A}, \boldsymbol{B}$, Targeting of PSD95-GFP-expressing PGNs for TEM analysis. $\boldsymbol{A}$, PSD95-GFP-labeled PGNs (circled in $\boldsymbol{A}$ ) were located in a fluorescent microscope under low resolution. A smaller area was then mechanically isolated (inset) and processed for anti-GFP immuno-TEM. Scale bar, $200 \mu \mathrm{m}$. $\boldsymbol{B}$, Electron micrograph of a labeled synapse from the isolated area shown in $\boldsymbol{A}$. The dark-grained aggregate in the magnified view (arrow) is located within a dendrite and is contacted by a presynaptic ORN axon terminal. Scale bars, $200 \mathrm{~nm}$. C, Confocal micrographs of a single glomerulus (single optical sections). Red, Labeling of an ORN-specific presynaptic marker (VGLUT2); green, a PSD95-GFP-expressing PGN (14 d.p.i.). The images on the right are magnifications from the dashed square regions in the left image. Note appositions (yellow; arrows) between presynaptic ORN terminals and postsynaptic PSD95-GFP puncta. Not all puncta were opposed to this presynaptic marker (green; arrowheads). Scale bar, $20 \mu \mathrm{m}$.

al., 2006). Together, we conclude that PSD95-GFP puncta serve as good proxy for putative synapses, in vivo (Niell et al., 2004).

To rule out the possibility that transgene expression impedes normal development, we exhausted a comparison between GFPexpressing neurons and PSD95-GFP-expressing neurons and found them to be similar in all the analyses we have performed. Specifically, GFP and PSD95-GFP neurons had similar dendritic morphologies, a similar morphological maturation pattern, and similar GC spine densities (corresponding to synaptic density; see supplemental Fig. $2 A-F$, available at www.jneurosci.org as supplemental material). PSD95-GFP expression did not seem to affect the life span of adultborn neurons because these could be even found 8-9 months after injection (supplemental Fig. 2G,H, available at www.jneurosci.org as supplemental material). These control experiments clearly show that expressing PSD95-GFP using lentivirus transduction does not considerably impede PGNs morphology, maturation, synaptic density, nor does it affect life longevity. Having established a method to tag putative synapses in vivo, we next followed the developmental dynamics of PSD95-GFP puncta by time-lapse in vivo two-photon imaging of immature and mature PGNs.

\section{PSD95-GFP dynamics of immature adult-born PGNs}

We imaged PSD95-GFP puncta of adult-born PGNs in anesthetized, freely breathing mice. In vivo two-photon imaging revealed 

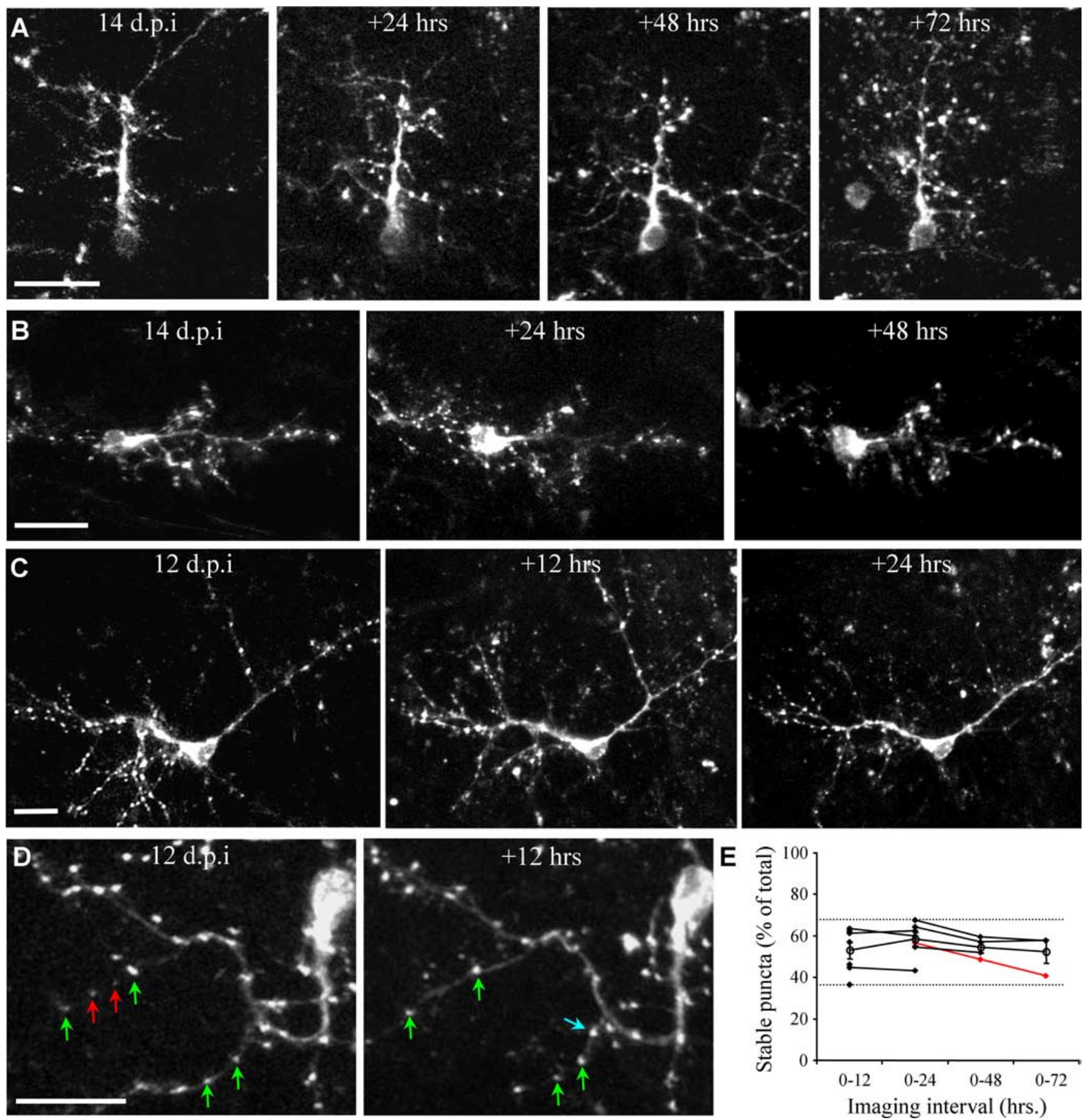

Figure 3. PSD95-GFP dynamics of immature adult-born PGNs at different imaging intervals. $\boldsymbol{A}-\boldsymbol{D}$, Projection images from consecutive in vivo imaging sessions $24 \mathrm{~h}$ apart $(\boldsymbol{A}, \boldsymbol{B})$ and $12 \mathrm{~h}$ apart. $\boldsymbol{C}, \boldsymbol{D}$, Examples of stable, lost, and new puncta are marked with green, red and blue arrows, respectively (same neuron as in supplemental Movie 1, available at www.jneurosci.org as supplemental material). $\boldsymbol{E}$, Quantitative analysis of puncta dynamics, at different imaging intervals. For clarity, only the percentage of stable puncta (out of the total number) is shown. Full range is indicated by the dashed lines, open circles indicate means ( $n=11$ neurons, from 6 mice). Isolated points (not connected to a line) are from neurons which were only imaged twice. Lines connect consecutive sessions from the same neuron. For example, the red line shows data of the neuron in $A$, which was imaged four times. The percentage of stable, new, and lost puncta were not significantly different between all imaging intervals (Kruskal-Wallis test; stable puncta: $p=0.32$, lost puncta: $p=0.38$, new puncta: $p=0.35$ ). Neurons imaged at 12,13 or $14 \mathrm{~d}$.p.i. had similar values of stable, lost, and new puncta. All values are mean \pm SEM. Scale bars, $20 \mu \mathrm{m}$.

high contrast, high resolution images of both dendrites and puncta (Figs. 1, 3-6). An example of a complete $Z$-stack of a PGN from a single in vivo imaging session is shown in supplemental Movie 1, available at www.jneurosci.org as supplemental material.

One advantage of using in vivo two-photon imaging is the ability to study structural dynamics over time. Indeed, multiple imaging sessions for long durations can reveal temporal dynamics at multiple time scales (Holtmaat et al., 2005). However, multiple imaging sessions suffer from increased likelihood to cause photodynamic damage, and long imaging intervals may result in uncontrolled effects in the underlying tissue. Therefore, we looked for the shortest imaging interval in which significant puncta dynamics occurred, and could be revealed using only two 
imaging sessions. We performed time-lapse imaging of 11 neurons in a total of 32 imaging sessions ( $n=6$ mice), ranging from four sessions at $24 \mathrm{~h}$ intervals down to two sessions at $12 \mathrm{~h}$ intervals (Fig. 3). As expected from immature neurons, both dendrites and puncta were highly dynamic. We never observed a completely stable neuron.

Puncta dynamics were analyzed on stable dendrites from consecutive imaging sessions. For example, Figure 3, A-D, shows neurons that were imaged 2-4 times at 12 or $24 \mathrm{~h}$ intervals. Each puncta was scored as either stable, new, or lost (see examples in Fig. $3 D$; green, blue and red arrows, respectively). At this immature developmental stage only $36-67 \%$ of PSD95-GFP puncta per neuron remained stable in consecutive imaging sessions (Fig. $3 E$, dashed lines) and the rest were either new or lost (for clarity, only data of stable puncta are shown in Fig. $3 E$ ). Stability was similar in 12, 24, 48 and $72 \mathrm{~h}$ imaging intervals. For example, during $72 \mathrm{~h}$ between imaging sessions, $52 \pm 6 \%$ (mean \pm SEM) of PSD95-GFP puncta were stable (Fig. 3E, $0-72$, open circle) $(n=3)$, and during $12 \mathrm{~h}$ between imaging sessions $53 \pm 4 \%$ were stable (Fig. 3E, $0-12$, open circle) $(n=7)$. These values were not significantly different across all imaging intervals tested (0-12, $0-24,0-48$ and $0-72$ h; Kruskal-Wallis Test, $p=0.32$ ). However, as expected, when examining different imaging intervals of the same neuron, stability usually decreased with increasing imaging intervals (6/7 neurons) (Fig. 3E, lines). Thus, PSD95-GFP puncta of immature neurons are in a highly dynamic state which can be revealed during a $12 \mathrm{~h}$ period; an interval which we continued to use throughout this study. We next asked whether synapses of mature neurons also remain dynamic. To answer this question, we analyzed mature ( $\sim 40$ d.p.i.) neurons by time-lapse imaging.

\section{Distribution and dynamics of PSD95-GFP puncta in mature adult-born PGNs}

We hypothesized that PSD95-GFP puncta of mature neurons would remain dynamic because recent work by our group demonstrated significant levels of dendritic dynamics in these cells (Mizrahi, 2007). To test this issue, we imaged mature PGNs ( $\sim 40$ d.p.i.) at $12 \mathrm{~h}$ intervals and compared their morphology and dynamics to those of immature PGNs (Fig. 4A,B). Compared with immature neurons, mature PGNs had a higher total dendritic branch length (TDBL), more branch points, and twice the number of puncta per neuron (Fig. $4 C-E$ ). In both mature and immature PGNs the average distance between PSD95-GFP puncta along dendrites remained similar, indicating that synapses and dendrites grow in parallel, at least up to this age (immature: $5.6 \pm 0.2 \mu \mathrm{m}$; mature: $5.4 \pm 0.1 \mu \mathrm{m}$; $t$ test, $p=0.22$ ).

The dynamic behavior of mature and immature PGNs was also different. Specifically, mature PGNs had 25\% more stable puncta (i.e., $\sim 75 \%$ of total) and $50 \%$ less new puncta (i.e., $\sim 13 \%$ of total) compared with immature PGNs (Fig. $4 F$ ). Thus, as adult-born neurons matured, PSD95-GFP puncta became more stable. Despite the higher stability, PSD95-GFP puncta still remained highly dynamic suggesting that PGNs continuously form and eliminate synapses, not only during early development but well after they have integrated into the network. We next tested whether local sensory input might play a role in the early development of adult-born PGN dendrites and synapses.

Sensory input regulates the development of adult-born PGNs Little is currently known about the mechanisms that determine developmental choices of adult-born neurons. Mechanisms of cell fate decisions are intracellular in nature and might already be
A

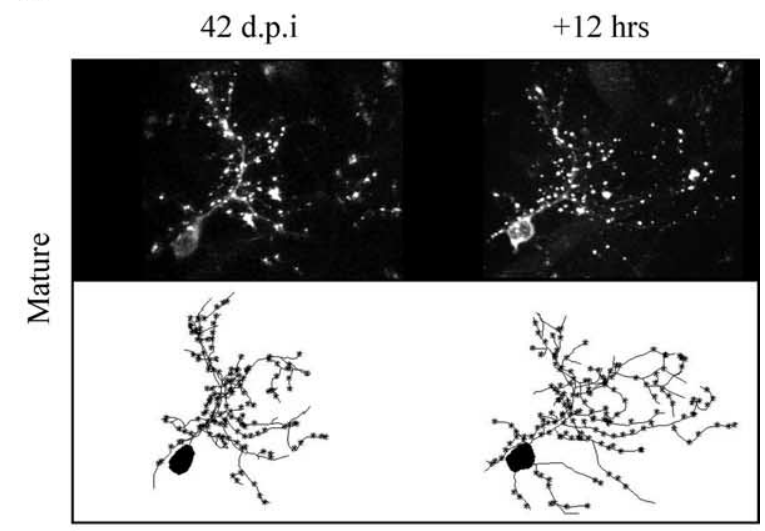

B

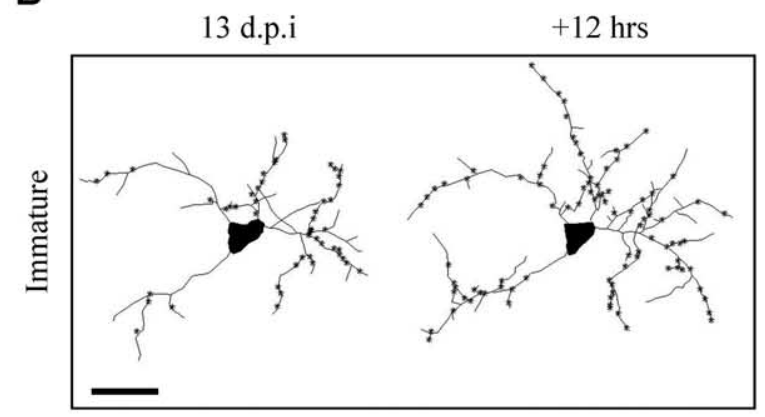

C

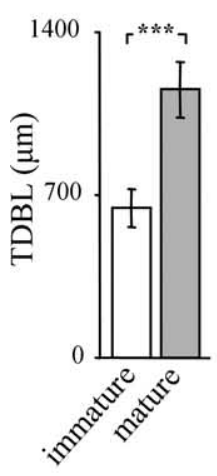

$\mathbf{F}$

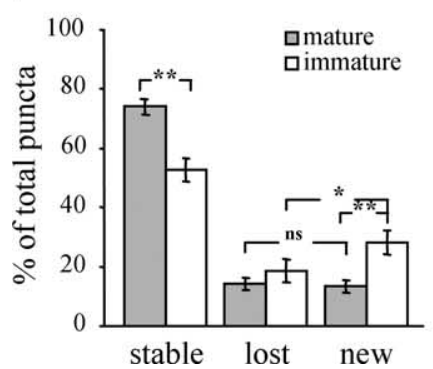

D

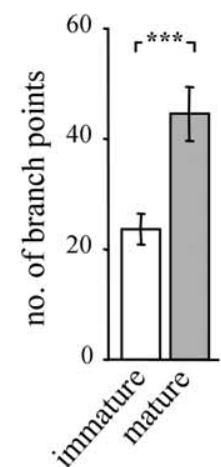

E
Figure 4. Maturation of adult-born PGNs: morphology and puncta dynamics. $A$, Time-lapse images of a mature adult-born PGN (42 d.p.i.). Top: Projections, bottom: reconstructions. Asterisks denote the location of PSD95-GFP puncta along the dendrite. $\boldsymbol{B}$, Reconstructions of time-lapse images of an immature adult-born PGN (13 d.p.i.). Scale bar, $20 \mu \mathrm{m}$. C $-\boldsymbol{E}$, Morphometric comparison of immature and mature adult-born PGNs. Bar graphs of the total dendritic branch length (TDBL) $(\boldsymbol{C})$, number of branch points $(\boldsymbol{D})$, and the total number of puncta per neuron $(\boldsymbol{E}) . n=10$ immature PGNs from 5 mice, and 7 mature PGNs from 6 mice. $\boldsymbol{F}$, Analysis of puncta dynamics. Mature PGNs had significantly more stable puncta and significantly less new puncta. Furthermore, immature PGNs had a significantly higher percentage of new puncta than lost. In contrast, mature PGNs had a similar percentage of new and lost puncta. $n=7$ immature PGNs from 4 mice, and 5 mature PGNs from 3 mice. All values are mean $\pm \mathrm{SEM}^{*} p<0.04,{ }^{* *} p<0.005,{ }^{* * *} p<0.001$ (Mann-Whitney test). 
determined during migration or even at the stem cell niche (Hack et al., 2005; Waclaw et al., 2006; Merkle et al., 2007). However, after neuronal fate is determined and neurons reach their target zone, what mechanisms guide their developmental and integration strategies? Driven by the observation of the high-levels of puncta dynamics (Fig. 3), we hypothesized that synaptogenesis within glomeruli is continuously regulated. One mechanism that might serve as a regulator of synaptogenesis is sensory input. PGNs are located perfectly to test this hypothesis because their dendrites are compactly arranged within glomeruli, which receive functionally homogeneous synaptic input from highly convergent axonal projections. To test this hypothesis, we combined intrinsic signal imaging (ISI) with in vivo two-photon imaging of immature PGNs.

The experimental design is schematically outlined in Figure $5 A$. For each mouse, we constructed an odor map of the dorsal surface of the OB using ISI (Fig. 5B). We used an odor composed of a low-concentration mixture of butanal and methylbenzoate ( $1: 1 ; 10$ ppm each) which activated numerous glomeruli in the mediolateral region of the dorsal surface (Fig. 5B). After odor maps were constructed, mice were immediately injected with lenti-PSD95-GFP in the SVZ and then returned to their home cages. Three to four days after injection mice were exposed to a small odor pot containing low quantities of the odor mixture (150 $\mu \mathrm{l}$ on a swab). Odor pots were placed in the home cage, replaced daily, and maintained until the end of the experiment (from 3-4 to 12-14 d.p.i.). We imaged a total of 17 PGNs ( $n=10$ mice), 9 of which were located in IS-active (enriched) domains and 8 in IS-nonactive (nonenriched) domains. A neuron was considered "within" an IS-active domain if its domain obeyed both threshold and temporal dynamics criteria of the ISI signal (see Materials and Methods and Fig. 5B for an example). PGNs outside sensory active domains were at least $0.5 \mathrm{~mm}$ away from the closest active glomerulus. In a separate control experiment we verified that the IS maps remained stable before and after enrichment, precluding the possibility of major physiological changes in ORN input to PGNs (supplemental Fig. 3, available at www. jneurosci.org as supplemental material).

A standard morphological analysis revealed that PGNs growing within sensory enriched loci were almost twice as large compared with PGNs in the nonenriched loci (Fig. 5C). This striking increase was evident in all tested morphological parameters such as TDBL, number of branch points, as well as in the number of puncta per neuron and the neurons' spatial territory coverage (i.e., Sholl analysis) (Fig. 5D-G; compare black and white bars and full lines). Moreover, sensory enrichment upregulated the morphology of enriched PGNs well out of the range of normal, immature, PGNs (Fig. 5D-G). Reconstructions of all the neurons from the enrichment experiments are shown in supplemental Figure 4, available at www.jneurosci.org as supplemental material. Interestingly, the morphological complexity of enriched neurons was upregulated to level of "mature" cells. We could not distinguish "enriched" (immature) PGNs from mature PGNs nor nonenriched from immature PGNs in naive mice (Fig. 5D-G; data from naive mice is shown again for comparison and is the same as in Fig. 4C-E, dashed gray bars and lines). Thus, sensory enrichment locally upregulated the morphology of immature PGNs to levels of mature PGNs.

\section{Dynamics of odor-enriched adult-born PGNs}

The pronounced increase in morphological complexity of enriched immature neurons, to the level of mature neurons, was evident by basic morphological analyses. However, to test whether enrichment changed their dynamics, we used time-lapse imaging. Dynamics during a $12 \mathrm{~h}$ interval was tested in 6 neurons, which were located within enriched domains. Time-lapse data from these cells was compared with the data collected in naive mice from both immature and mature PGNs (same dataset shown Fig. $4 F$ ). Examples of time-lapse sequences from the three different groups are shown in Figure 6A-C. Although enriched neurons had the morphological complexity of mature neurons, they still showed dynamic behavior reminiscent of immature PGNs. Specifically, the percentage of stable puncta in enriched neurons was significantly smaller than that of mature PGNs from naive mice, and not significantly different from that of immature PGNs from naive mice (Fig. 6D). This result suggests that sensory enrichment does not simply accelerate the normal growth pattern of adult-born PGNs.

Another hallmark of immature neuronal dynamics is that they had significantly more new puncta than lost puncta; a difference which was no longer evident at maturity (Fig. $4 F$ ). The larger fraction of new puncta indicates that immature PGNs are in a process of overall growth. In this respect, enriched PGNs resembled mature neurons because the levels of new and lost puncta were also not significantly different (Fig. 6E). Thus, on one hand, enrichment accelerated the development of immature PGNs such that the overall number of potential synapses grew, but overall growth stopped. However, enrichment did not change the overall structural stability of the PGNs indicating that neuronal age rather than size sets the levels of structural plasticity.

\section{Discussion}

We describe a system for imaging putative synapses of adult-born PGNs, in vivo. Using time-lapse two-photon imaging, we showed that synaptic puncta are dynamic, suggesting that synaptogenesis is continuously regulated. Using a combined structural and functional imaging approach we showed that development of adultborn PGNs is locally upregulated by sensory activity.

\section{Sensory input regulates the development of adult-born neurons}

Neuroblasts from the SVZ migrate through the RMS and into the OB where they become fully functional interneurons. The fate of new neurons is already determined at the SVZ by their birth place and not by the host environment (Kelsch et al., 2007; Merkle et al., 2007). Moreover, the distinct location within the SVZ will determine the expression pattern of specific transcription factors (TFs), which in turn determine neuronal fate (Hack et al., 2005; Waclaw et al., 2006). If "fate" is depicted as the anatomical location and immunocytochemical characterization of the cell, then the PGNs that we described were already fated. However, once fated and in place, what shapes PGNs' local growth, synaptic integration and connectivity pattern formation? Our data show that one regulator is sensory input.

Sensory input has been classically recognized as important in shaping development and plasticity throughout the nervous system (Katz and Shatz, 1996; Sanes and Lichtman, 2001; Nithianantharajah and Hannan, 2006). In the mouse OB, sensory input has been studied during development and in adulthood by a variety of manipulations ranging from odor deprivation to genetic silencing of ORNs (Brunjes, 1994; Zhao and Reed, 2001). Sensory input plays a regulatory role during initial wiring of the glomeruli (Kerr and Belluscio, 2006) as well as in competitive interactions between ORNs as they re-innervate existing glomeruli in adulthood (Zheng et al., 2000). The majority of activitydependent manipulations are based on substantial perturbation 
A

2.

Lenti-PSD95-GFP
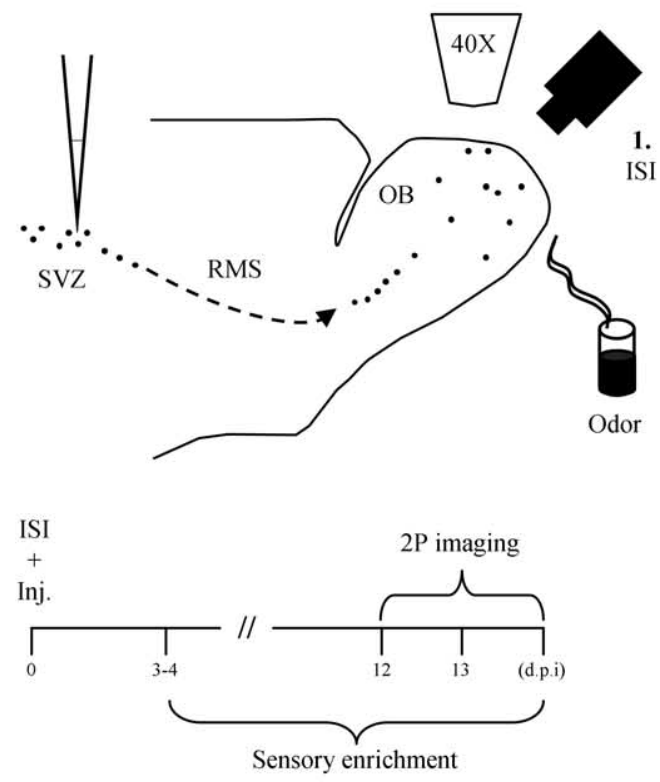

C non-enriched

(ROI 1)
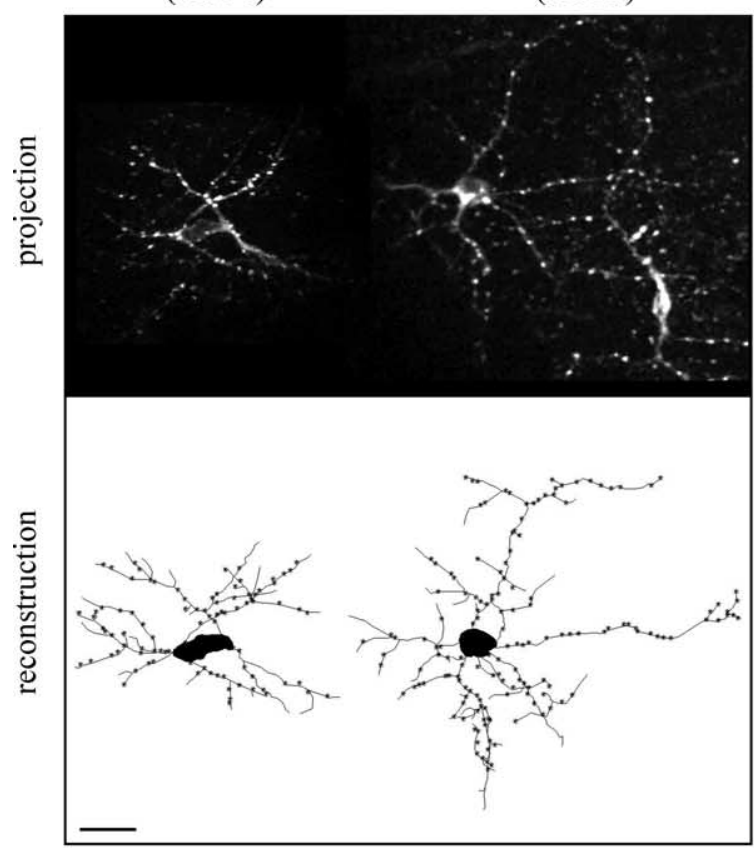

B
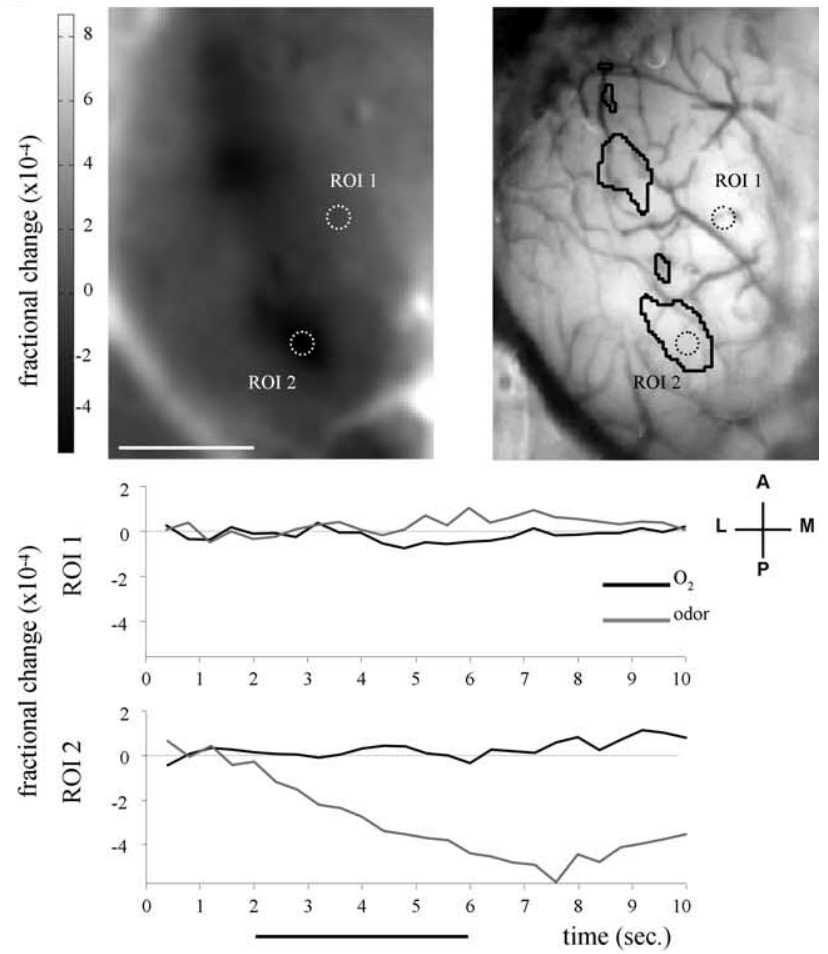

D

E
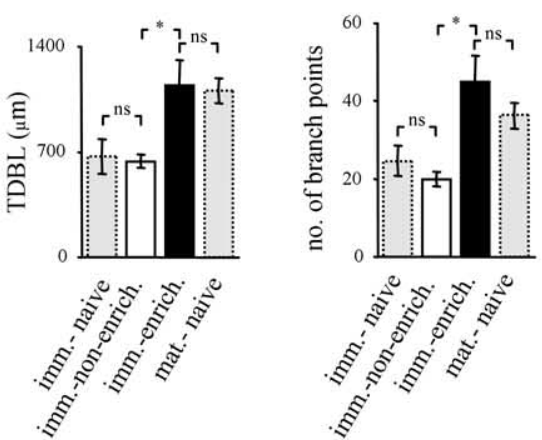

$\mathbf{F}$
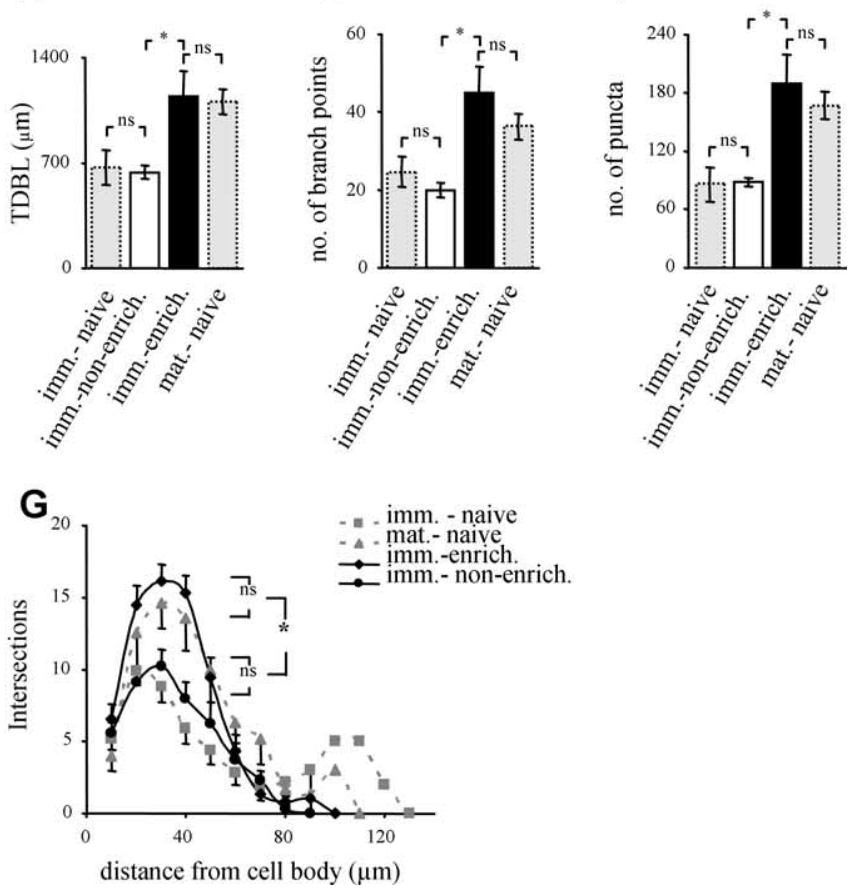

Figure 5. Local sensory input upregulates adult-born PGN development. $A$, Schematic representation of the experimental protocol (top) and time course of the ISI-targeted 2 P imaging experiment (bottom). Inj., Lentivirus injection into the SVZ. B, C, A representative ISI-2P experiment. B, Odor map (top left), and blood vessel map (top right), of the intrinsic signal response to the odor mixture (IS-active domains are circled in blackin the blood vessel map). Two regions of interest (ROIS), containing the neurons shown in C, are circled in both maps. ROI 1 is in an IS-nonactive domain, whereas ROI 2 is within an IS-active domain. Bottom, The intrinsic signal time course of a single trial in ROIs 1 and 2. Notice the intrinsic signal response in ROI 2, but no response in ROI 1. Black trace, Pure oxygen; gray trace, odor. Horizontal black line shows the stimulus duration (4s). A, Anterior; P, posterior; L, lateral; M, medial; scale bar, $1 \mathrm{~mm}$. C, Two adult-born PGNs from the same experiment shown in $B$, one from a nonenriched domain (IS-nonactive, ROI 1 ) and another from an enriched domain (IS-active, ROI2). Top, Maximum projection images of the originalZ-stacks. Bottom, Two-dimensional view of the reconstructed neurons at the top. Scale bar, $20 \mu \mathrm{m}$. $\mathbf{D}$ - $\mathbf{G}$, Quantitative morphological comparisons of neurons from enriched domains and nonenriched domains (black and white bars, respectively). Neurons from enriched domains had significantly greater total dendritic branch length (TDBL) $(\boldsymbol{D})$, number of branch points $(\boldsymbol{E})$, and number of puncta per neuron $(\boldsymbol{F}) . \boldsymbol{G}$, Sholl analysis, showing that the significant differences between neurons in enriched domains versus those in nonenriched domains, were centered between $20-50 \mu \mathrm{m}$ from the cell body. All higher values of enriched neurons were similar to those of mature neurons from naive mice (dashed gray bars and lines). The lowervalues of neurons from nonenriched domains were similar to those of immature neurons from naive mice (dashed gray bars and lines). $n=9 \mathrm{PGNs}$ in enriched domains and $n=8 \mathrm{PGN}$ s in nonenriched domains. Morphological data of immature and mature neurons from naive mice are the same as in Figure 4 , and are presented here as dashed gray bars and lines. All values are mean \pm SEM, ${ }^{*} p<0.001$ (Kruskal-Wallis test, followed by Mann-Whitney test). Imm.-naive, Immature PGNs from naive mice; mat.-naive, mature PGNs from naive mice; imm.-enrich., immature PGNs from enriched domains in enriched mice; imm.-nonenrich., immature PGNs from nonenriched domains in enriched mice. 

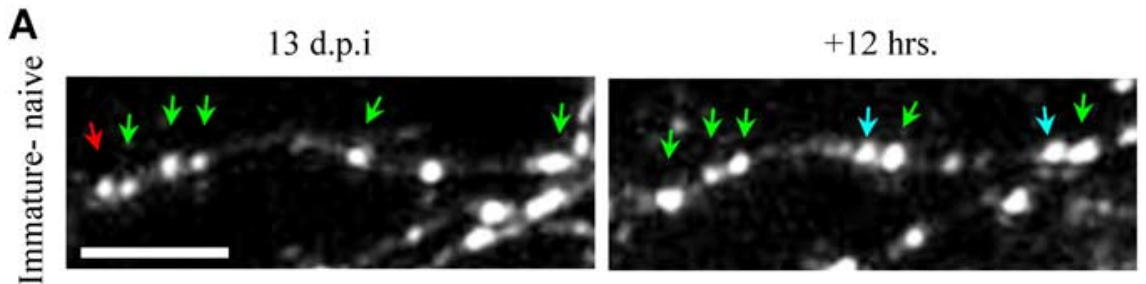

B
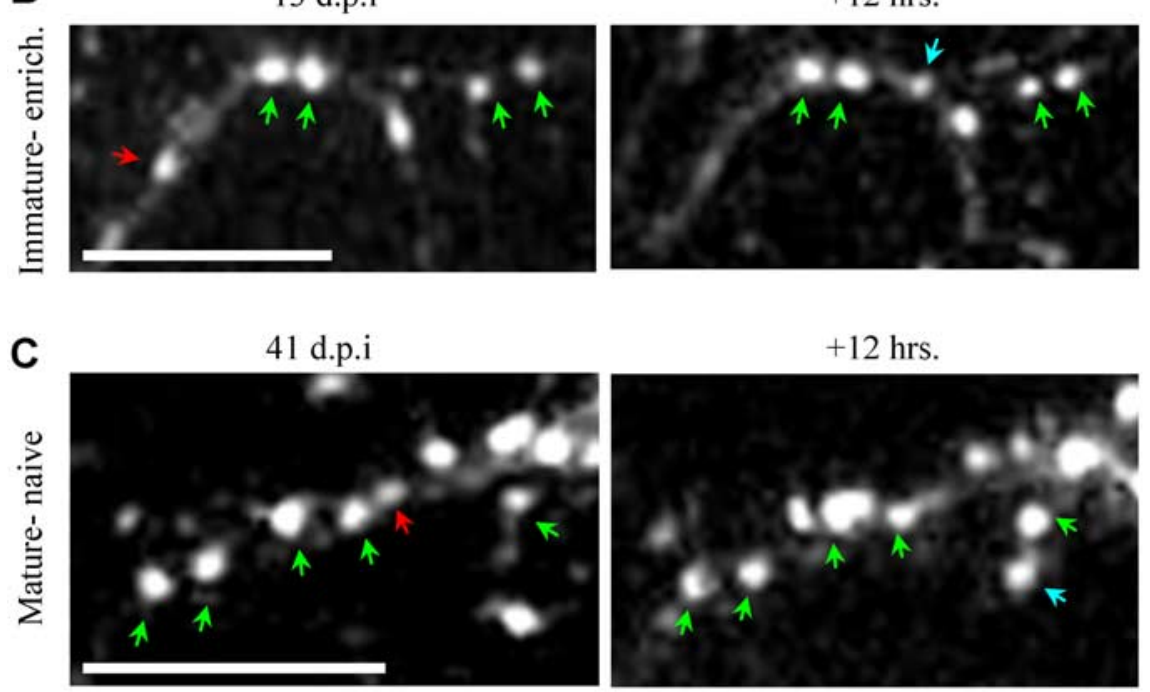

D

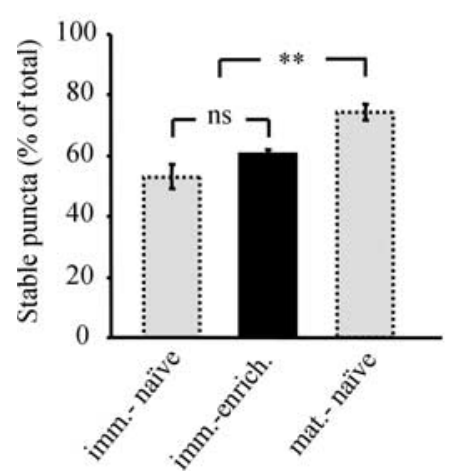

\section{E}

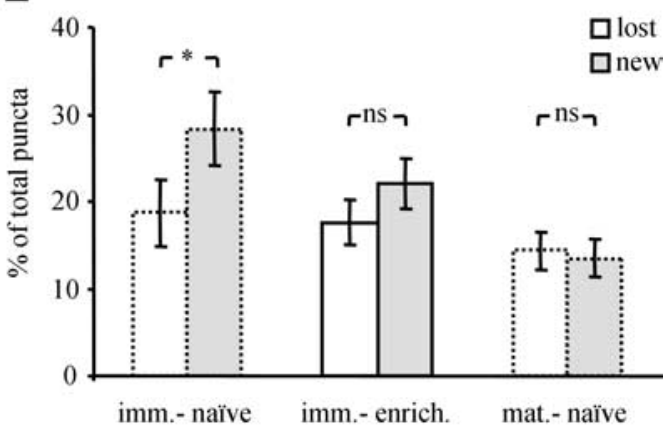

Figure 6. Dynamics of odor-enriched adult-born PGNs compared with immature and mature adult-born PGNs. $\boldsymbol{A}-\boldsymbol{C}$, Images of PSD95-GFP puncta from $12 \mathrm{~h}$ time-lapse sequences, in vivo. Immature PGN from a naive mouse $(\boldsymbol{A})$, an immature PGN from an enriched domain $(\boldsymbol{B})$, and a mature PGN from a naive mouse (C). Examples of stable, lost, and new puncta are marked with green, red and blue arrows, respectively. D, Bar graphs showing the levels of stable puncta which is higher in mature PGNs. E, Bar graphs of puncta dynamics. In contrast to immature PGNs from naive mice, mature PGNs and immature PGNs from enriched domains had similar values of new and lost puncta. $n=5$ immature PGNs from enriched domains, from 3 mice. All values are mean $\pm S E M$, ${ }^{*} p<0.04,{ }^{* *} p<0.003$ (Kruskal-Wallis test and Mann-Whitney test). Note that data of puncta dynamics of immature and mature neurons from naive mice is the same as in Figure 4 (dotted bars). Scale bars, $10 \mu \mathrm{m}$. Imm.-naive, Immature PGNs from naive mice; mat.-naive, mature PGNs from naive mice; imm.-enrich., immature PGNs from enriched domains in enriched mice.

of the system, leading to compensatory responses in the existing circuitry. For example, nostril occlusion during postnatal development not only affected the survival and morphology of adultborn GCs, but also triggered compensatory responses in resident GCs (Saghatelyan et al., 2005).

In previous work by our group we also used unilateral nostril occlusion in adults and found no significant effects on dendritic morphology and dynamics of adult-born PGNs, after short deprivation periods (Mizrahi, 2007). Those data suggested that nat- urally occurring spontaneous and sensory-evoked activity of ORNs does not play a significant role in early stages of the development of those PGNs. Indeed, naturally occurring sensory-evoked activity of ORNs, in naive animals, may vary considerably and is thus difficult to control experimentally. In this work, we considerably improved our control over the sensory manipulations by targeting active glomeruli which responded to odor presentation, and nonactive regions which did not. Simple odor exposure significantly upregulated morphological development and synaptogenesis of PGNs, only in sensory enriched glomeruli, and not in others. Although our results were significant across animals, the strongest evidence comes from neurons growing in the same tissue, some of which were separated only by several hundred micrometers (Fig. 5C).

Our data suggest that local sensory input induces an increase in the numbers of potential synapses during the time of integration into the network. Although the nature of the extra synapses is not known, comparison of the dynamics in enriched PGNs vs immature and mature PGNs reveals some insight about the effects of the sensory enrichment. On one hand, enrichment induced more puncta and caused net growth to cease, similar to mature PGNs. One the other hand, the overall puncta population was not as stable as in mature PGNs (this is schematically shown in Fig. 7). We propose a model in which the age of the neuron (enriched or not) determines its dynamic state, but the overall neuron size and number of synapses have an upper bound limit.

Our dataset is limited to postsynaptic dynamics. These postsynaptic dynamics might also reflect presynaptic dynamics of the PGNs' presynaptic partners (i.e., ORNs, Mitral/Tufted Cells, and possibly centrifugal inputs). This is especially relevant to ORN inputs, which continuously turnover in the olfactory epithelium throughout life. Thus, as ORN axons constantly break and form synaptic connections they are expected to influence the postsynaptic dynamics of adult-born PGNs as well. In contrast, Mitral cells have been shown to be structurally stable over long periods (Mizrahi and Katz, 2003), and should have a minor affect on postsynaptic PGN dynamics compared with ORN axons. Whether stabilization of synapses is effected by the age and structural stability of its presynaptic partner is not known but it is clear that adult-born neurons can form synapses with preexisting presynapses within the circuit (Toni et al., 2007).

The cellular mechanism by which sensory enrichment works might be via increasing stability of newborn synapses and thereby causing the neuron to lose less synapses. Consequently, this in- 
crease in the number of synapses, may promote an increased growth or stabilization of new dendrites (Cline and Haas, 2008). Alternatively, sensory enrichment may promote increased dendritic growth (concomitant with synapse formation on new dendrites); thereby increasing the total amount of stable, new and lost synapses, while keeping the overall turnover rate constant. Either mechanism might be achieved by means of direct synaptic activity, but perhaps also by activity-dependant secretion of neurotrophic factors by ORN axons, similar to the regulation of axonal competition within glomeruli (Cao et al., 2007). Data from the hippocampus, showed that molecular components like GABA and NMDA receptors play a role in activity-dependent regulation during synaptic integration of adult-born neurons (Ge et al., 2006; Tashiro et al., 2006), a mechanism which might well be shared by adult-born neurons in the OB.

Behaviorally induced plasticity in the mammalian $\mathrm{OB}$ was addressed with respect to survival rates of adult-born neurons (Rochefort et al., 2002; Magavi et al., 2005; Alonso et al., 2006). Alonso et al. (2006) showed that survival rates do not change after mere sensory activation, but only after learning. Although we did not study survival rates, our data show that mere local sensory activation does increase synaptogenesis. It is difficult to directly compare the results between the studies because different methods have been used to detect sensory active regions. We used ISI whereas others used immunohistochemistry of immediate early genes and genetic targeting as proxy for locating sensory active domains. Both strategies have advantages and disadvantages and it would be interesting to investigate whether and how learning-induced activation shapes the different stages of adult neurogenesis using ISI as the targeting approach. Notably, we did not use odor exposure in a specific behavioral context. Rather, we used simple odor exposure to promote local activation in the OB. Whether and how different levels of odor stimulation will affect adult-born development remains to be seen. In simpler systems like Drosophila, it has been recently proposed that the local olfactory interneurons (corresponding to the mammalian $\mathrm{OB}$ interneurons) may be the basis for experience-dependent plasticity, induced by prolonged exposure to an odorant (Sachse et al., 2007).

\section{Synaptic integration and connectivity pattern of adult-born PGNs}

Adult neurogenesis has been largely studied at the level of proliferation, migration, and survival but much less is known about synaptic integration and consequent connectivity patterns (Lledo et al., 2006; Toni et al., 2007; Whitman and Greer, 2007a). Specifically, who are the presynaptic partners impinging on the adult-born PGNs? And in turn, which neurons receive input from the PGNs? Although the latter question remains completely open, the former question has been partially addressed. For example, patch clamp recordings from adult-born PGNs showed the existence of ORN $\rightarrow$ PGN synapses (Belluzzi et al., 2003; Grubb et al., 2008). In accordance with these data, in targeted EM
Immature

(2 weeks)
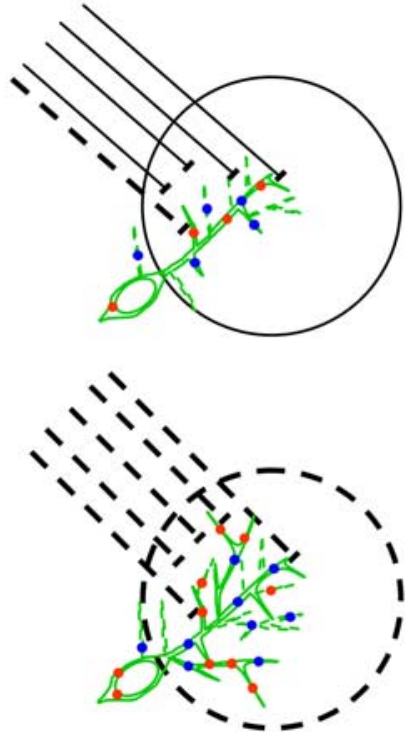

Mature
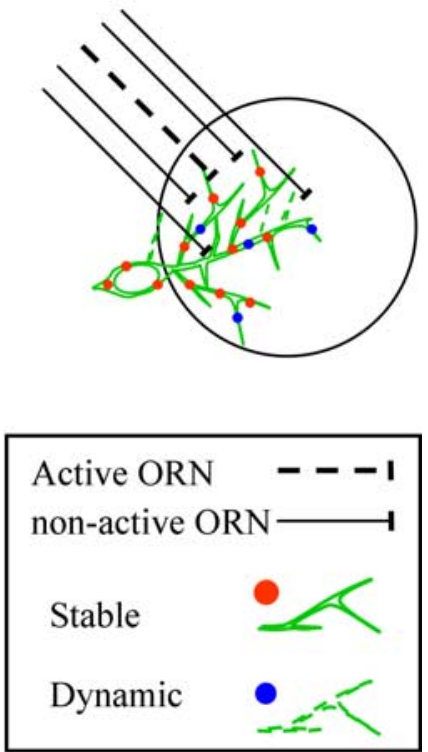

(7 weeks)

Figure 7. Schematic model of the developmental upregulation induced by sensory enrichment. $\boldsymbol{A}$, Under animal facility conditions, most glomeruli will not be robustly activated by the odor environment of the animal (notice only few active ORNs). induce highly robust neuronal activity from $0 R$ Rs in select glomeruli. Under these conditions, dendritic and synaptic dynamics are changed resulting in an increase of the total numbers of dendrites and synapses. See Discussion.

screens we always (14/14 PGNs, $8.7 \pm 1.3$ synapses from each $\mathrm{PGN}$ ) found $\mathrm{ORN} \rightarrow \mathrm{PGN}$ synapses (see supplemental Fig. 5, available at www.jneurosci.org as supplemental material). Furthermore, almost all adult-born PGNs examined with light microscopy, had PSD95-GFP puncta within ORN-zones (20/21) (Fig. 2C), supporting the presence of an ORN $\rightarrow$ PGN synapse in adult-born PGNs.

This connectivity pattern suggests that the adult-born PGNs that we studied here might be "type I" neurons as determined by current nomenclature, based on synaptic connectivity and immunocytochemical markers (Kosaka and Kosaka, 2007). However, further immunocytochemical analysis is needed to verify this. One reservation from this interpretation concerns possible variability resulting from the variability of injection sites between different studies (Merkle et al., 2007) and species and strainspecificity (Kosaka and Kosaka, 2007). In rats, ORN $\rightarrow$ PGN synapse is the exception rather the rule (Hayar et al., 2004; Wachowiak and Shipley, 2006). In addition, EM data from BALB/C mice (the strain used here), found no evidence for ORN $\rightarrow$ PGN synapses (White, 1972, 1973). The rarity of ORN $\rightarrow$ PGN synapses in general sampling techniques versus their abundance in targeted analyses from different injection sites (Belluzzi et al., 2003; Grubb et al., 2008) suggests that this synapse might hold special importance for adult-born PGNs.

\section{References}

Alonso M, Viollet C, Gabellec MM, Meas-Yedid V, Olivo-Marin JC, Lledo PM (2006) Olfactory discrimination learning increases the survival of adult-born neurons in the olfactory bulb. J Neurosci 26:10508-10513.

Altman J (1969) Autoradiographic and histological studies of postnatal neurogenesis. 3. Dating the time of production and onset of differentiation of cerebellar microneurons in rats. J Comp Neurol 136:269-293.

Alvarez-Buylla A, Garcia-Verdugo JM (2002) Neurogenesis in adult subventricular zone. J Neurosci 22:629-634.

Bagley J, LaRocca G, Jimenez DA, Urban NN (2007) Adult neurogenesis 
and specific replacement of interneuron subtypes in the mouse main olfactory bulb. BMC Neurosci 8:92.

Belenky MA, Smeraski CA, Provencio I, Sollars PJ, Pickard GE (2003) Melanopsin retinal ganglion cells receive bipolar and amacrine cell synapses. J Comp Neurol 460:380-393.

Belluzzi O, Benedusi M, Ackman J, LoTurco JJ (2003) Electrophysiological differentiation of new neurons in the olfactory bulb. J Neurosci 23:10411-10418.

Bozza T, Feinstein P, Zheng C, Mombaerts P (2002) Odorant receptor expression defines functional units in the mouse olfactory system. J Neurosci 22:3033-3043.

Brunjes PC (1994) Unilateral naris closure and olfactory system development. Brain Res Brain Res Rev 19:146-160.

Cao L, Dhilla A, Mukai J, Blazeski R, Lodovichi C, Mason CA, Gogos JA (2007) Genetic modulation of BDNF signaling affects the outcome of axonal competition in vivo. Curr Biol 17:911-921.

Carlén M, Cassidy RM, Brismar H, Smith GA, Enquist LW, Frisén J (2002) Functional integration of adult-born neurons. Curr Biol 12:606-608.

Carleton A, Petreanu LT, Lansford R, Alvarez-Buylla A, Lledo PM (2003) Becoming a new neuron in the adult olfactory bulb. Nat Neurosci 6:507-518.

Cline HT, Haas K (2008) The regulation of dendritic arbor development and plasticity by glutamatergic synaptic input: a review of the synaptotrophic hypothesis. J Physiol 586:1509-1517.

Ebihara T, Kawabata I, Usui S, Sobue K, Okabe S (2003) Synchronized formation and remodeling of postsynaptic densities: long-term visualization of hippocampal neurons expressing postsynaptic density proteins tagged with green fluorescent protein. J Neurosci 23:2170-2181.

Feng G, Mellor RH, Bernstein M, Keller-Peck C, Nguyen QT, Wallace M, Nerbonne JM, Lichtman JW, Sanes JR (2000) Imaging neuronal subsets in transgenic mice expressing multiple spectral variants of GFP. Neuron 28:41-51.

Gabellec MM, Panzanelli P, Sassoè-Pognetto M, Lledo PM (2007) Synapsespecific localization of vesicular glutamate transporters in the rat olfactory bulb. Eur J Neurosci 25:1373-1383.

Ge S, Goh EL, Sailor KA, Kitabatake Y, Ming GL, Song H (2006) GABA regulates synaptic integration of newly generated neurons in the adult brain. Nature 439:589-593.

Grubb MS, Nissant A, Murray K, Lledo PM (2008) Functional maturation of the first synapse in olfaction: development and adult neurogenesis. J Neurosci 28:2919-2932.

Hack MA, Saghatelyan A, de Chevigny A, Pfeifer A, Ashery-Padan R, Lledo PM, Götz M (2005) Neuronal fate determinants of adult olfactory bulb neurogenesis. Nat Neurosci 8:865-872.

Hayar A, Karnup S, Ennis M, Shipley MT (2004) External tufted cells: a major excitatory element that coordinates glomerular activity. J Neurosci 24:6676-6685.

Holtmaat AJ, Trachtenberg JT, Wilbrecht L, Shepherd GM, Zhang X, Knott GW, Svoboda K (2005) Transient and persistent dendritic spines in the neocortex in vivo. Neuron 45:279-291.

Katz LC, Shatz CJ (1996) Synaptic activity and the construction of cortical circuits. Science 274:1133-1138.

Kelsch W, Mosley CP, Lin CW, Lois C (2007) Distinct mammalian precursors are committed to generate neurons with defined dendritic projection patterns. PLoS Biol 5:e300.

Kerr MA, Belluscio L (2006) Olfactory experience accelerates glomerular refinement in the mammalian olfactory bulb. Nat Neurosci 9:484-486.

Knott GW, Holtmaat A, Wilbrecht L, Welker E, Svoboda K (2006) Spine growth precedes synapse formation in the adult neocortex in vivo. Nat Neurosci 9:1117-1124.

Kosaka K, Kosaka T (2007) Chemical properties of type 1 and type 2 periglomerular cells in the mouse olfactory bulb are different from those in the rat olfactory bulb. Brain Res 1167:42-55.

Lledo PM, Alonso M, Grubb MS (2006) Adult neurogenesis and functional plasticity in neuronal circuits. Nat Rev Neurosci 7:179-193.

Magavi SS, Mitchell BD, Szentirmai O, Carter BS, Macklis JD (2005) Adultborn and preexisting olfactory granule neurons undergo distinct experience-dependent modifications of their olfactory responses in vivo. J Neurosci 25:10729-10739.

Merkle FT, Mirzadeh Z, Alvarez-Buylla A (2007) Mosaic organization of neural stem cells in the adult brain. Science 317:381-384.

Micheva KD, Smith SJ (2007) Array tomography: a new tool for imaging the molecular architecture and ultrastructure of neural circuits. Neuron 55:25-36.

Mizrahi A (2007) Dendritic development and plasticity of adult-born neurons in the mouse olfactory bulb. Nat Neurosci 10:444-452.

Mizrahi A, Katz LC (2003) Dendritic stability in the adult olfactory bulb. Nat Neurosci 6:1201-1207.

Niell CM, Meyer MP, Smith SJ (2004) In vivo imaging of synapse formation on a growing dendritic arbor. Nat Neurosci 7:254-260.

Nithianantharajah J, Hannan AJ (2006) Enriched environments, experience-dependent plasticity and disorders of the nervous system. Nat Rev Neurosci 7:697-709.

Petreanu L, Alvarez-Buylla A (2002) Maturation and death of adult-born olfactory bulb granule neurons: role of olfaction. J Neurosci 22:6106-6113.

Rochefort C, Gheusi G, Vincent JD, Lledo PM (2002) Enriched odor exposure increases the number of newborn neurons in the adult olfactory bulb and improves odor memory. J Neurosci 22:2679-2689.

Sachse S, Rueckert E, Keller A, Okada R, Tanaka NK, Ito K, Vosshall LB (2007) Activity-dependent plasticity in an olfactory circuit. Neuron $56: 838-850$.

Saghatelyan A, Roux P, Migliore M, Rochefort C, Desmaisons D, Charneau P, Shepherd GM, Lledo PM (2005) Activity-dependent adjustments of the inhibitory network in the olfactory bulb following early postnatal deprivation. Neuron 46:103-116.

Sanes JR, Lichtman JW (2001) Induction, assembly, maturation and maintenance of a postsynaptic apparatus. Nat Rev Neurosci 2:791-805.

Sholl DA (1953) Dendritic organization in the neurons of the visual and motor cortices of the cat. J Anat 87:387-406.

Tashiro A, Sandler VM, Toni N, Zhao C, Gage FH (2006) NMDA-receptormediated, cell-specific integration of new neurons in adult dentate gyrus. Nature 442:929-933.

Toni N, Teng EM, Bushong EA, Aimone JB, Zhao C, Consiglio A, van Praag H, Martone ME, Ellisman MH, Gage FH (2007) Synapse formation on neurons born in the adult hippocampus. Nat Neurosci 10:727-734.

Wachowiak M, Shipley MT (2006) Coding and synaptic processing of sensory information in the glomerular layer of the olfactory bulb. Semin Cell Dev Biol 17:411-423.

Waclaw RR, Allen ZJ 2nd, Bell SM, Erdélyi F, Szabó G, Potter SS, Campbell K (2006) The zinc finger transcription factor Sp8 regulates the generation and diversity of olfactory bulb interneurons. Neuron 49:503-516.

Washbourne P, Bennett JE, McAllister AK (2002) Rapid recruitment of NMDA receptor transport packets to nascent synapses. Nat Neurosci 5:751-759.

White EL (1972) Synaptic organization in the olfactory glomerulus of the mouse. Brain Res 37:69-80.

White EL (1973) Synaptic organization of the mammalian olfactory glomerulus: new findings including an intraspecific variation. Brain Res 60:299-313.

Whitman MC, Greer CA (2007a) Synaptic integration of adult-generated olfactory bulb granule cells: basal axodendritic centrifugal input precedes apical dendrodendritic local circuits. J Neurosci 27:9951-9961.

Whitman MC, Greer CA (2007b) Adult-generated neurons exhibit diverse developmental fates. Dev Neurobiol 67:1079-1093.

Xu HT, Pan F, Yang G, Gan WB (2007) Choice of cranial window type for in vivo imaging affects dendritic spine turnover in the cortex. Nat Neurosci 10:549-551.

Zhao H, Reed RR (2001) X inactivation of the OCNC1 channel gene reveals a role for activity-dependent competition in the olfactory system. Cell 104:651-660.

Zheng C, Feinstein P, Bozza T, Rodriguez I, Mombaerts P (2000) Peripheral olfactory projections are differentially affected in mice deficient in a cyclic nucleotide-gated channel subunit. Neuron 26:81-91. 